\title{
From concept to publication: Celebrating mentorship and knowledge generation through research during postgraduate residency training
}

\author{
W. Scott Beattie, MD • François Donati, MD, PhD • \\ Davy Cheng, MD • David Mazer, MD • \\ Donald R. Miller, MD
}

Received: 4 April 2013/Accepted: 17 April 2013/Published online: 27 April 2013

(c) Canadian Anesthesiologists' Society 2013

The aspiration to support and foster the conduct of highquality research is at the very core of our specialty. Part of the Canadian Anesthesiologists' Society (CAS) mission, as stated in 1947, is "to advance the art and science of anesthesia". A Research is our future. Every academic health sciences centre department of anesthesiology not only recognizes this fact but also endorses the conduct of highquality research, as does the Association of Canadian University Departments of Anesthesia (ACUDA). An important objective of ACUDA is to "promote the development of research and scientific progress in anesthesiology." B In our view, acquisition of robust skills in research methodology and scientific writing is an essential part of our specialty's

Electronic supplementary material The online version of this article (doi:10.1007/s12630-013-9955-1) contains supplementary material, which is available to authorized users.

W. S. Beattie, MD $(\bowtie) \cdot$ D. Mazer, MD

Department of Anesthesiology, Toronto General Hospital,

University of Toronto, 200 Elizabeth Street, Toronto,

ON M5G 2C4, Canada

e-mail: scott.beattie@uhn.on.ca

F. Donati, MD, PhD

Department of Anesthesiology, University of Montreal,

Montreal, QC, Canada

D. Cheng, MD

Department of Anesthesia and Perioperative Medicine,

Western University, London, ON, Canada

D. R. Miller, MD

Department of Anesthesiology, University of Ottawa,

Ottawa, ON, Canada mission to advance the knowledge base that forms the scientific foundation of patient care. This knowhow is particularly important for trainees who are streaming into academic career paths as clinician researchers or clinician investigators.

A centrepiece of the CAS Annual Meeting is the Resident Research Competition, established in 1967 by a generous grant from Wyeth Laboratories, Inc. Some 20 years earlier (in 1947), the CAS established a committee on research and applied (unsuccessfully) for funding the following year from the National Research Council (NRC) Canada. The Resident Research Competition has a vast legacy, being the first tangible research support offered by the Society. The Competition preceded, by 18 years, the establishment of the Canadian Anesthetists' Society Research Award (as it was called at the time) in 1985. The Resident Research Competition has been a regular feature of the CAS Annual Meeting since its inception, and past participants represent a "who's who" of anesthesia in Canada and internationally. A list of past awardees is available as electronic supplementary material online at: http://www.springer.com/12630. Many of these former residents became involved in prolific academic careers and had a strong impact on the specialty. Many of their achievements have been documented previously by Dr. C.D. Mazer and Dr. K. McClenaghan who reported the

\footnotetext{
A Shephard DA. Excerpted from Watching Closely Those Who Sleep, 1993. Available through the Canadian Anesthesiologists' Society Central office.

B ACUDA. Available from URL: http://www.cas.ca/english/acudaconstitution (accessed March 2013).
} 
results of a survey of 226 former residents who presented at the Competition during the years 1969-94. ${ }^{1}$ Applications for the Resident Research Competition are invited by way of submitted abstracts from postgraduate year 1 (PGY1) to postgraduate year 5 (PGY5) resident physicians enrolled in any one of the 17 Canadian university departments of anesthesia. Applications are reviewed by the CAS Annual Meeting Abstracts Committee. The top six applications advance to the Residents' Oral Competition and are judged by an ACUDA research representative from each university. Vitaid Canada Inc. has sponsored yearly donations to the Canadian Anesthesia Research Foundation (CARF) to support what is currently referred to as the "CAS/LMAVitaid Residents' Research Award". The Vitaid Award, which is not linked to the Residents' Research Competition, provides $\$ 10,000$ of operating grant support and is adjudicated by the CAS Research Advisory Committee together with all other CAS research grant award applications.

We recognize that the Royal College of Physicians and Surgeons of Canada (RCPSC) specialty training requirements for anesthesia encourage up to one year of research training in the Scholar domain of the CanMEDS competency of a residency program. ${ }^{\mathrm{C}}$ Nevertheless, with an extensive national curriculum to cover and new restrictions for hours of on-call duty, many anesthesiology program directors struggle to provide protected research time while respecting clinical training requirements. Our residency programs are also challenged by a limited number of faculty research mentors and resources to conduct research, both at the bench and at the bedside. Despite these challenges, over the years a number of anesthesia residents have become very involved in research projects and training in clinical epidemiology during residency. To showcase some of the excellent work being presented at the Resident Research Competition, all six residents who presented in the 2012 competition were invited by the Journal's editorial team to prepare manuscripts and to submit their work for peer review in consideration for publication. In this issue, we present the work from four of these young investigators (two others submitted their work elsewhere). The breadth, diversity, and clinical relevance of these articles are impressive. The contributors are to be congratulated for their responsiveness during the peer review process, which provided a unique opportunity for most to navigate the admittedly complex editorial peer review system for the first time and to experience the excitement of publication.

In one of these reports, Dr. G. Kostandoff et al. from McMaster University conducted a retrospective case

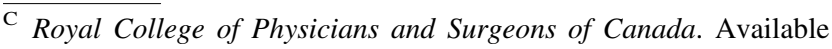
from URL: http://rcpsc.medical.org/residency/certification/training/ anesthesiology_e.pdf (accessed March 2013).
}

control study of 400 random procedures specifically to search for risk factors for unplanned admission after ambulatory surgery. ${ }^{2}$ Unplanned admission after outpatient surgery is an adverse event adding inconvenience and costs. Several risk factors were identified in this analysis as being highly predictive of unplanned admissions, including age, body mass index, and American Society of Anesthesiologists class III or higher. All of these situations are known before surgery, and a prospective assessment utilizing these risk factors is now required.

The second resident research article relates to a growing concern surrounding postoperative confusion states and delirium. This is especially problematic after major cardiorespiratory procedures, and it is hypothesized that this condition is associated with cerebral hypoxia. Dr. R. Brinkman et al. from the University of Manitoba conducted a prospective observational study monitoring cerebral oximetry in thoracic surgery patients. $^{3}$ Study results showed that every patient experienced cerebral desaturation when converted to one-lung ventilation. Furthermore, one quarter of the patients had extreme cerebral desaturation despite normal peripheral oxygen saturation. While they were unable to elucidate a mechanism for this finding, the ramifications may be clinically groundbreaking. Clearly, more studies are required to correlate these findings with meaningful outcomes and to assess effective preventive and treatment strategies.

The third resident article in this issue evaluates sternal wound infections, which remain a serious complication in cardiac surgery. Several small and poorly risk-adjusted studies have associated sternal wound infection with the need for tracheostomy. In a study that would be almost impossible to conduct within a randomized trial, Dr. L. Sun et al. from the University of Ottawa conducted a retrospective review of the institutional cardiac surgical database. Sophisticated analysis was used to risk adjust the patient populations to account for the obvious biases in this type of analysis, and the study results suggest that, even in the highest risk patients, there is a $50 \%$ increase in the odds of a sternal wound infection when patients have received a tracheostomy. ${ }^{4}$

The fourth article considers ultrasound imaging in perioperative medicine. The use of transesophageal echocardiography (TEE) is now almost universal in cardiac surgery and is gaining popularity in vascular surgical patients with a high risk of cardiac events. Dr. A. Gregory et al. from the University of Calgary reports on the utility of TEE to calculate aortic compliance and ventricular mechanics using relatively new "speckle tracking" software in combination with a blood pressure trace. ${ }^{5}$ This is a further demonstration of the increasing potential for ultrasound in clinical practice. It is also obvious that further research is required before this methodology can be 
employed; however, this study shows feasibility in the clinical context.

Research during postgraduate training is often an inadequately recognized endeavour that requires a commitment well beyond the scope of clinical training. Any research study requires a detailed protocol that should be registered and have a funding source, ethical approval, and resources for recruitment, data management, and statistical analysis. After presentation of the abstract, the final results need to be written and submitted as a manuscript, which usually requires two to three revisions prior to final acceptance. The process from concept development to publication can take several years. Is it any surprise that some residents are hesitant to even begin the process? The Society continues to highlight these residents-in-training at the CAS Annual Meeting, and we encourage the Annual Meeting planners to highlight this legacy by not scheduling competing sessions during the residents' competition. We should do more. Importantly, we encourage the ACUDA residency training programs to emphasize and facilitate a period of 612 months of research training as scholarly activity for a greater number of trainees. From the Journal's perspective, we shall continue to foster the training of future investigators and encourage residents to submit their work for peer review in consideration for publication. We offer Journal Electives as an introduction to editorial peer review as well as a Resident Observership Program. We welcome feedback from our readers regarding these and other initiatives to provide meaningful opportunities for Canadian residents to participate in research during their postgraduate training.

\section{Du concept à la publication: une célébration du mentorat et de la génération des connaissances grâce à la recherche pendant la résidence}

Au cœur même de notre spécialité se trouve le désir de soutenir et d'encourager la réalisation de recherches de grande qualité. Une partie de la mission de la Société canadienne des anesthésiologistes (SCA), énoncée en 1947 déjà, est «de faire progresser l'art et la science de l'anesthésie ». ${ }^{\mathrm{A}} \mathrm{La}$ recherche est notre avenir. Non

\footnotetext{
A Shephard DA. Excerpted from Watching Closely Those Who Sleep, 1993. Available through the Canadian Anesthesiologists' Society Central office.
}

seulement chaque département universitaire d'anesthésiologie reconnait ce fait, mais il soutient la réalisation de recherches de qualité élevée, tout comme le fait l'Association canadienne universitaire des départements d'anesthésie (ACUDA). Un objectif important de l'ACUDA est de «promouvoir le développement de la recherche et des progrès scientifiques en anesthésiologie. $»^{\mathrm{B}}$ À notre avis, l'acquisition de compétences solides en méthodologie de recherche et en rédaction scientifique est une partie essentielle de la mission de notre spécialité. Ces compétences permettent de faire progresser la base de connaissances qui constitue la fondation des soins aux patients. Ce savoir-faire est particulièrement important pour les résidents qui se lancent sur les sentiers d'une carrière universitaire en tant que chercheurs cliniciens.

Une importante composante du Congrès annuel de la SCA est le Concours de recherche des résidents, créé en 1967 grâce à une généreuse bourse des Laboratoires Wyeth, Inc. Quelque 20 ans plus tôt (en 1947), la SCA avait créé un comité sur la recherche et postulé (sans succès) pour obtenir l'année suivante du financement de la part du Conseil national de recherches Canada (CNRC). Le Concours de recherche des résidents possède un riche héritage, étant le premier fonds de soutien à la recherche tangible offert par la Société. Le Concours précédait de 18 ans la création, en 1985, de la Bourse de recherche de la Société canadienne des anesthésistes (comme on l'appelait à l'époque). Depuis sa création, le Concours de recherche des résidents revient régulièrement comme composante du Congrès annuel de la SCA, et les participants des années passées constituent un véritable 'bottin mondain' de l'anesthésie au Canada et dans le monde. Une liste des récipiendaires passés est disponible en ligne sous forme de matériel électronique supplémentaire à l'adresse: http://www.springer.com/12630. Bon nombre de ces anciens résidents se sont engagés dans des carrières universitaires prolifiques et ont eu un impact important sur la spécialité. Plusieurs de leurs réalisations ont été documentées par le passé par les Dr C.D. Mazer et Dr K. McClenaghan, qui ont rapporté les résultats d'un sondage réalisé auprès de 226 anciens résidents qui avaient présenté leurs travaux au Concours entre 1969 et $1994 .{ }^{1}$ Les soumissions pour le Concours de recherche des résidents sont reçues sous forme de résumés soumis par les médecins résidents de la première à la cinquième année (R1-R5) inscrits dans l'un des 17 départements d'anesthésie des universités canadiennes. Les soumissions sont passées en revue par le Comité des résumés du Congrès annuel de la SCA. Les six meilleures soumissions passent au prochain tour, le Concours oral des résidents, où elles sont évaluées par un représentant de la

\footnotetext{
B ACUDA. Available from URL: http://www.cas.ca/english/acudaconstitution (accessed March 2013).
} 
recherche de l'ACUDA de chaque université. Vitaid Canada Inc. a offert des contributions financières annuelles à la Fondation canadienne de recherche en anesthésie (FCRA) afin de financer le prix actuellement nommé « Bourse de recherche SCA/LMA Vitaid pour les résidents ». La Bourse Vitaid, qui n'est pas liée au Concours de recherche des résidents, offre $10000 \$$ de subvention de fonctionnement et est adjugée par le Comité consultatif de la recherche de la SCA, comme toutes les autres soumissions pour des bourses de recherche de la SCA.

Nous sommes conscients que les exigences de la formation spécialisée en anesthésie du Collège royal des médecins et chirurgiens du Canada (CRMCC) encouragent jusqu'à une année de formation en recherche dans le cadre de la section «érudition» des compétences CanMEDS d'un programme de résidence. ${ }^{\mathrm{C}}$ Cependant, en raison d'un vaste curriculum national à couvrir et des nouvelles restrictions concernant les heures de garde, plusieurs directeurs de programme d'anesthésiologie ont peine à garantir du temps de recherche protégé à leurs résidents tout en respectant les exigences de formation clinique. Nos programmes de résidence sont également mis à mal par un nombre limité de mentors de recherche et de ressources pour réaliser des recherches, en laboratoire ou au chevet des patients. Malgré ces défis, au fil des ans plusieurs résidents en anesthésie se sont engagés très activement dans des projets de recherche et des formations en épidémiologie clinique au cours de leur résidence. Afin de présenter certains des excellents travaux soumis dans le cadre du Concours de recherche des résidents, l'équipe de rédaction du Journal a invité les six résidents finalistes du concours de 2012 à préparer des manuscrits et à soumettre leur article à la révision par les pairs en vue d'une possible publication. Dans ce numéro, nous présentons les travaux de quatre de ces jeunes chercheurs (deux autres ont soumis leurs travaux ailleurs). L'étendue, la diversité et la pertinence clinique de ces articles sont impressionnantes. Il faut féliciter les contributeurs pour leur réceptivité pendant le processus de révision par les pairs, qui leur a offert une occasion unique de naviguer les méandres complexes du système de révision par les pairs pour la première fois et de ressentir la fébrilité qui précède la publication.

Dans l'un de ces comptes rendus, Dr G. Kostandoff $e t$ coll., de l'Université McMaster, ont réalisé une étude cas témoins rétrospective portant sur 400 interventions prises au hasard pour déterminer spécifiquement s'il existait des facteurs de risque d'admission imprévue après une chirurgie ambulatoire. ${ }^{2}$ L'admission imprévue après une chirurgie ambulatoire est un événement fâcheux qui

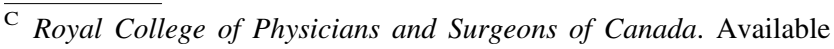
from URL: http://rcpsc.medical.org/residency/certification/training/ anesthesiology_e.pdf (accessed March 2013).
}

entraîne inconvénients et coûts supplémentaires. Plusieurs facteurs de risque ont été identifiés dans cette analyse comme étant de bons prédicteurs d'admissions imprévues, notamment l'âge, l'indice de masse corporelle et un statut de classe III ou plus selon la classification de l'American Society of Anesthesiologists. Tous ces éléments sont connus avant la chirurgie, et une évaluation prospective se fondant sur ces facteurs de risque est désormais de mise.

Le deuxième article de recherche par un résident traite de l'inquiétude croissante autour des états de confusion et de délirium postopératoires. Ces états sont particulièrement problématiques après des interventions cardiorespiratoires majeures, et l'hypothèse que cette condition est associée à une hypoxie cérébrale est émise. Dr R. Brinkman et coll. de l'Université du Manitoba ont réalisé une étude observationnelle prospective qui monitorait l'oxymétrie cérébrale chez les patients subissant une chirurgie thoracique. ${ }^{3}$ Les résultats de l'étude ont montré que tous les patients ont subi une désaturation cérébrale lorsqu'ils ont été mis sous ventilation sélective. En outre, un quart des patients souffrait de désaturation cérébrale extrême malgré une saturation périphérique en oxygène normale. Bien que les auteurs n'aient pas pu déterminer le mécanisme sous-jacent à cette découverte, les ramifications pourraient être révolutionnaires d'un point de vue clinique. Il est évident que des recherches supplémentaires sont nécessaires pour corréler ces résultats à d'autres résultats significatifs et pour évaluer diverses stratégies efficaces de prévention et de traitement.

Le troisième article de résident dans ce numéro évalue les infections de plaie sternale, une complication toujours grave de la chirurgie cardiaque. Plusieurs études de petite envergure et mal ajustées pour le risque ont associé l'infection de plaie sternale au recours à la trachéostomie. Dans une étude qu'il serait quasi impossible de réaliser dans le cadre d'un essai randomisé, Dr L. Sun et coll. de l'Université d'Ottawa ont passé en revue, de façon rétrospective, la base de données de chirurgie cardiaque de leur centre. Des analyses élaborées ont été utilisées pour ajuster le risque des populations de patients afin de tenir compte des biais évidents de ce type d'analyse. Les résultats de l'étude suggèrent que même chez les patients les plus à risque, on observe une augmentation de $50 \%$ de la probabilité d'infection de plaie sternale lorsque les patients ont subi une trachéostomie. ${ }^{4}$

Le quatrième article porte sur l'échographie dans la médecine périopératoire. L'utilisation de l'échocardiographie transœsophagienne (ÉTO) est désormais quasi universelle en chirurgie cardiaque et gagne en popularité pour la prise en charge des patients de chirurgie vasculaire présentant un risque élevé de complications cardiaques. Dr A. Gregory et coll., de l'Université de Calgary, décrivent l'utilité de l'ÉTO pour calculer la compliance aortique et la 
mécanique ventriculaire à l'aide d'un logiciel de 'suivi de pixel' relativement nouveau, combiné à un tracé de la tension artérielle. ${ }^{5}$ Il s'agit ici d'une autre démonstration du potentiel grandissant de l'échographie dans la pratique clinique. Il est également évident que des recherches supplémentaires seront nécessaires avant que cette méthodologie ne puisse être employée; toutefois, cette étude montre la faisabilité d'une telle application dans le contexte clinique.

Pendant la formation postgraduée, la recherche est souvent une entreprise peu reconnue qui nécessite un engagement qui va bien au-delà des objectifs déclarés de la formation clinique. Toute étude nécessite un protocole détaillé, lequel doit être enregistré et disposer d'une source de financement, d'un consentement éthique, ainsi que de ressources pour le recrutement, la gestion des données et l'analyse statistique. Après présentation du résumé, les résultats finaux doivent être rédigés puis soumis sous forme de manuscrit, ce qui nécessite en général deux à trois révisions avant l'acceptation finale. Le processus entre la mise au point d'un concept et la publication peut prendre plusieurs années. Est-il si surprenant que certains résidents hésitent avant même de se lancer dans un tel processus? La Société continue de célébrer ces résidents en formation lors du Congrès annuel de la SCA, et nous encourageons les planificateurs du Congrès annuel à célébrer cet héritage en ne programmant pas d'autres séances simultanément au Concours des résidents. Nous devrions faire davantage. De manière importante, nous encourageons les programmes de formation en résidence de l'ACUDA à insister sur l'importance d'une période de 6-12 mois pour la formation en recherche en tant qu'activité d'érudition pour un plus grand nombre de résidents et en faciliter la mise en ouvre.
En ce qui touche au Journal, nous continuerons à encourager la formation de nos futurs chercheurs et invitons les résidents à soumettre leurs travaux à la révision par les pairs en vue d'une possible publication. Nous offrons aux résidents un Stage facultatif au Journal comme introduction à l'évaluation par les pairs ainsi qu'un Programme d'observateur. Nous invitons nos lecteurs à nous faire parvenir leurs commentaires concernant ces initiatives et d'autres qui visent à offrir des occasions intéressantes aux résidents canadiens de participer à la recherche pendant leur formation postgraduée.

Competing interests None declared.

Conflits d'intérêt Aucun.

\section{References}

1. Mazer CD, McClenaghan K. The CAS Residents' Competition: A 25 year review. Can J Anaesth 1994; 41: 116-9.

2. Whippey A, Kostandoff G, Paul J, Ma J, Thabane L, Ma HK. Predictors of unanticipated admission following ambulatory surgery: a retrospective case-control study. Can J Anesth 2013; 60: this issue. DOI: 10.1007/s12630-013-9935-5.

3. Brinkman R, Amadeo RJ, Funk DJ, Girling LG, Grocott HP, Mutch WA. Cerebral oxygen desaturation during one-lung ventilation: correlation with hemodynamic variables. Can J Anesth 2013; 60: this issue. DOI: 10.1007/s12630-013-9954-2.

4. Sun L, Boodhwan M, Baer H, McDonald B. The association between tracheostomy and sternal wound infection in postoperative cardiac surgery patients. Can J Anesth 2013; 60: this issue. DOI: 10.1007/s12630-013-9950-6.

5. Gregory AJ, Dobson G. Proximal aortic compliance and diastolic function assessed by speckle tracking imaging. Can J Anesth 2013; 60: this issue. DOI: 10.1007/s12630-013-9934-6. 